\title{
Experimental characterization of an angle-multiplexed holographic memory
}

\author{
Xin An and Demetri Psaltis \\ Department of Electrical Engineering, Mail Stop 116-81, California Institute of Technology, Pasadena, California 91125
}

Received May 24, 1995

\begin{abstract}
We demonstrate a simple angle-multiplexing holographic storage system, using a single acousto-optic deflector to achieve fast random access to the stored holograms. We used this system to store as many as 300 holograms in a $90^{\circ}$-geometry $\mathrm{Fe}: \mathrm{LiNbO}_{3}$ crystal. To characterize the system performance, we analyzed the reconstructions in terms of the signal-to-noise ratio. (c) 1995 Optical Society of America
\end{abstract}

Holographic storage of a large number of images by use of angle multiplexing was recently demonstrated. ${ }^{1,2}$ Acoustic-optic devices ${ }^{3-5}$ (AOD's) can be used to perform the angular scanning of the reference beam. AOD's offer several advantages over mechanical scanners, such as rapid access and accurate repeatable setting of the reference beam angle. In this Letter we utilize an architecture similar to that described in Refs. 3 and 5 to measure experimentally the fidelity of the reconstructions in an angle-multiplexed memory. We show that normalization of the reconstructions significantly improves the performance by suppressing deterministic sources of errors such as beam nonuniformity and multiple reflections in the crystal. Since such deterministic error sources can in principle be eliminated by careful engineering, the performance that we obtain through normalization provides an estimate for the performance that is expected from an optimized system. Moreover, the normalization technique we describe can be a useful tool for improving the performance of practical optical memories.

The system diagram of the experimental apparatus is shown in Fig. 1. We use a slow-shear $\mathrm{Te}_{2} \mathrm{O}_{3}$ AOD with a $42 \mathrm{~mm} \times 8 \mathrm{~mm}$ aperture. In the signal arm we use a 5:1 imaging system to illuminate the spatial light modulator (SLM; Epson liquid-crystal television device, $480 \times 440$ pixels in an area $27 \mathrm{~mm} \times$ $20 \mathrm{~mm}$ ). A second imaging system transfers the page displayed on the SLM onto a cooled CCD $(752 \times 480$ pixels) whose video output is digitized and transferred to the computer memory for evaluation. $\mathrm{A} \mathrm{LiNbO}_{3}$ crystal $\left(2 \mathrm{~cm} \times 1 \mathrm{~cm} \times 1 \mathrm{~cm}, 45^{\circ}\right.$ cut $)$ is placed just beyond the Fourier-transform plane at the center of this second imaging system. In the reference arm a $1: 1$ imaging system is used to image the AOD aperture onto the crystal so that the two beams interact at right angles.

The angular selectivity of this system is $\delta \theta=\lambda / A \approx$ $0.0035^{\circ}$, where $\lambda=488 \mathrm{~nm}$ and $A=8 \mathrm{~mm}$ is the diameter of the illuminated region. The relationship between the deflection angle outside the AOD and the change in the $\mathrm{rf}$ frequency is $\delta \theta=\lambda \Delta f / V_{s}$, where $\Delta f$ is the frequency change and $V_{s}$ is the acoustic velocity in the AOD. Therefore the minimum frequency distance between two neighboring holograms is approximately $75 \mathrm{kHz}$. The bandwidth of the AOD is $30 \mathrm{MHz}$, and therefore the maximum number of holograms that we can angularly multiplex in this system is 400 .
The measured angular selectivity of a recorded chessboard pattern is near $80 \mathrm{kHz}$, as shown in Fig. 2. Using $80 \mathrm{kHz}$ as the frequency spacing, we stored a total of 300 holograms. Eleven of these holograms were chessboard patterns used for measuring the performance of the reconstructions (evenly distributed in the recording sequence), another eleven were all-ones patterns used to normalize the rest of the holograms, and the rest were random-bit patterns. Figure 3 shows the diffration efficiencies of the 300 holograms. The maximum deviation of the diffraction efficiency from the mean value is less than $15 \%$.

The pixels on the SLM do not have a one-to-one correspondence with the pixels on the CCD. Therefore the 11 chessboard patterns consisting of large on and off blocks that can be easily delineated after detection are convenient to use to evaluate the fidelity of the reconstructions. We consider the signal detected on each CCD pixel as a separate sample that is to be classified as either on (bright) or off (dark). Each block of the chessboard pattern occupies $75 \times 70$ pixels on the CCD. A computer is programmed to select each block separately and classify all the pixels within the block. Approximately $80 \%$ of the pixels are discarded at the boundaries between the chessboard blocks to avoid crossover pixels. Following this procedure, we collected 126,000 on samples and the same number of off samples from each of the 11 chessboard patterns. These data were then used to characterize the peformance. We used the following definition of signal-

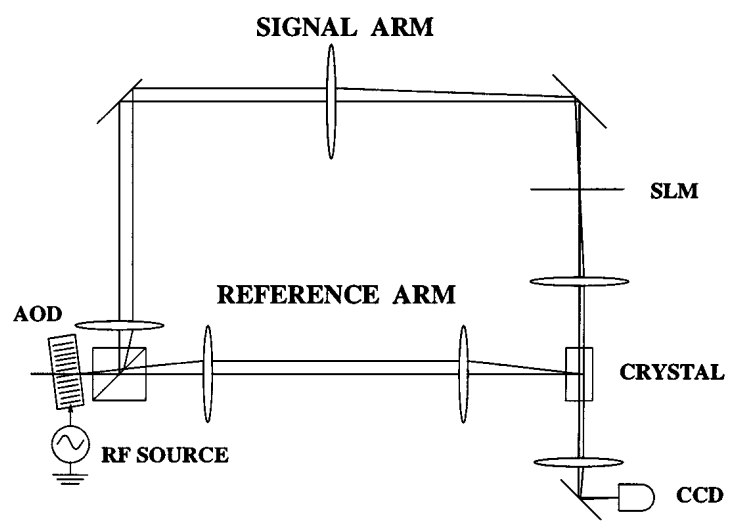

Fig. 1. System diagram. 


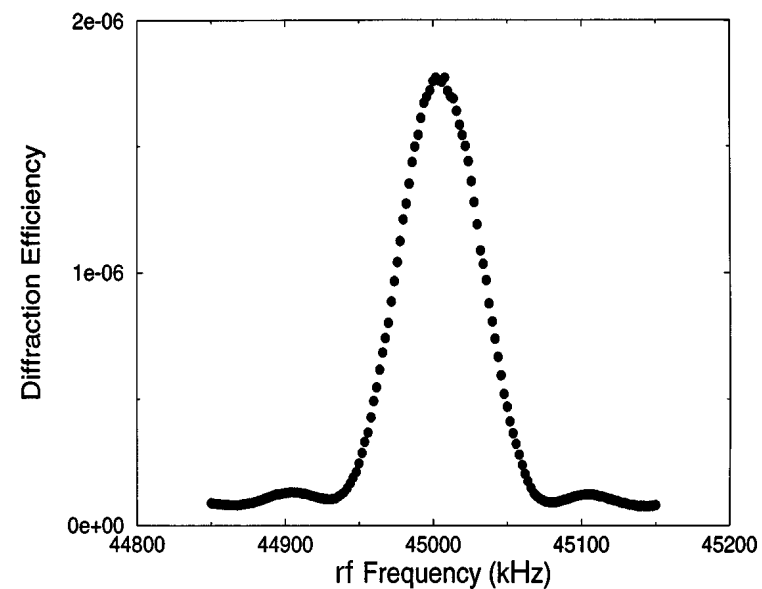

Fig. 2. Angular selectivity.

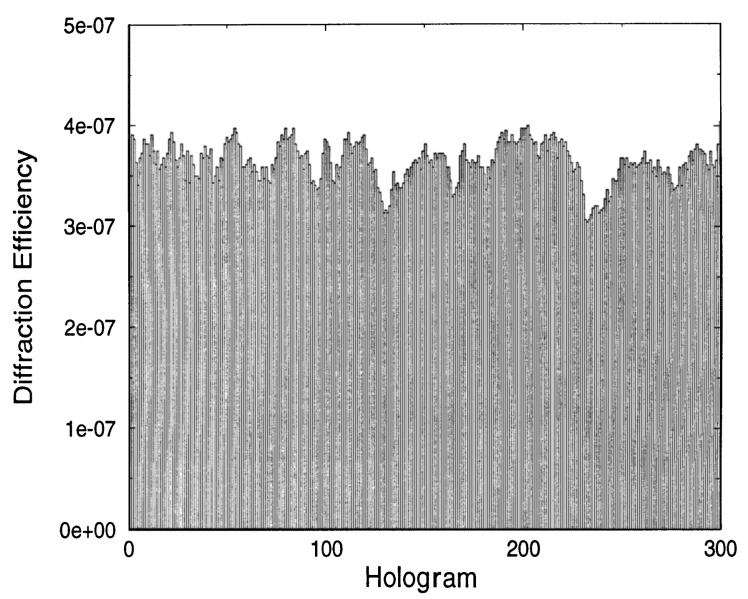

Fig. 3. Diffraction efficiencies of the 300 holograms stored in the system.

to-noise ratio (SNR) to assess the relative quality of images:

$$
\mathrm{SNR}=\frac{\mu_{1}-\mu_{0}}{\sqrt{\sigma_{1}^{2}+\sigma_{0}^{2}}},
$$

where $\mu_{0}$ and $\mu_{1}$ are the means of the off and on signals, respectively, and $\sigma_{0}^{2}$ and $\sigma_{1}^{2}$ are the corresponding variances.

To identify the sources of SNR degradation, we made a sequence of measurements under different conditions. The results are summarized in Fig. 4, in which the SNR is plotted on the vertical axis. The SNR's of the original reconstructions as detected on the CCD are indicated by the filled diamonds. The SNR degraded from 4.13 for the pattern on the SLM that was simply imaged to the output, to 3.82 after the crystal was inserted in the path of the signal beam, to 3.54 when the reconstruction of a single hologram was evaluated, and finally to an average SNR of 2.90 when all 300 holograms were recorded. This relatively small degradation implies that any noise introduced by the crystal or the holographic recording process is dominated by other sources of SNR degradation such as nonuniformities in the illumination and the SLM.

The SNR is significantly improved when the images are normalized. These measurements are indicated by the filled circles in Fig. 4 . Next to each of the 11 stored chessboard patterns an all-white page (all pixels on) was stored and reconstructed to represent the illumination profile. After inversion, normalization, and smoothing of these profile data, we scaled each pixel of the reconstructed chessboard image by the corresponding profile data point. In Fig. 4 the average SNR jumped from 2.90 to 8.03 after the compensation. In Fig. 5 the original reconstruction of one of the stored patterns is contrasted with the normalized version of the same pattern. For the normalized images there is a more pronounced difference between the SNR of the various test images and that obtained when all 300 holograms are stored. The SNR obtained in the absence of the crystal is limited principally by residual nonuniformity in the illumination and the SLM. The introduction of the crystal reduces the SNR because of uncompensated fringes owing to multiple reflections in the uncoated crystal, surface defects, and scatter noise. The additional reduction in SNR when a single hologram is stored is attributed to the nonuniformity of the reference beam and the spatially varying modulation depth. We significantly reduced the variation in the modulation depth by recording the holograms away from the Fourier plane. Finally, the SNR dropped to 8.03 when the entire sequence was recorded. The reason for the final drop is not the loss in signal strength, because the single hologram was recorded to have the same diffraction efficiency as each of the 300 holograms. Instead, we attribute it to three factors: cross talk (adjacent holograms were

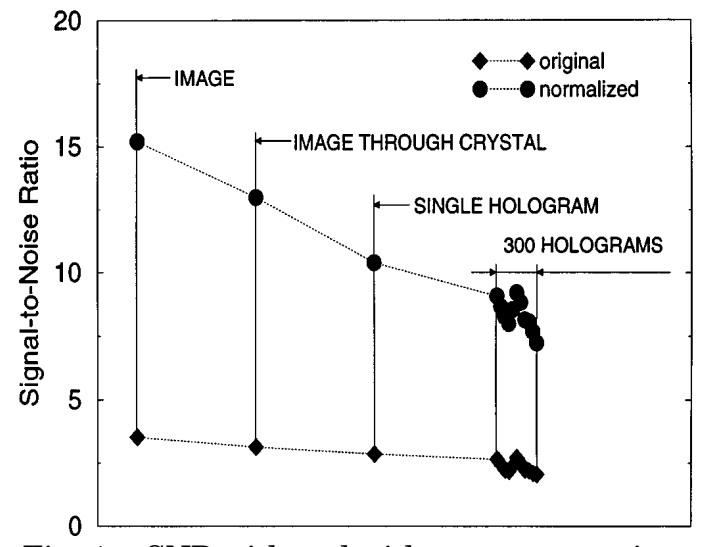

Fig. 4. SNR with and without compensation.

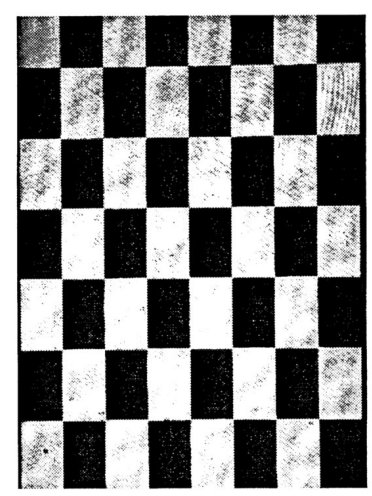

(a)

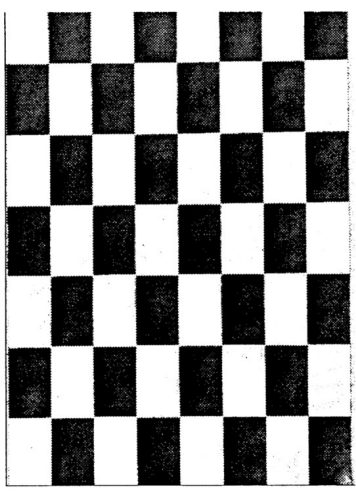

(b)
Fig. 5. (a) Original and (b) compensated reconstructions of hologram 180 of the 300 holograms. 


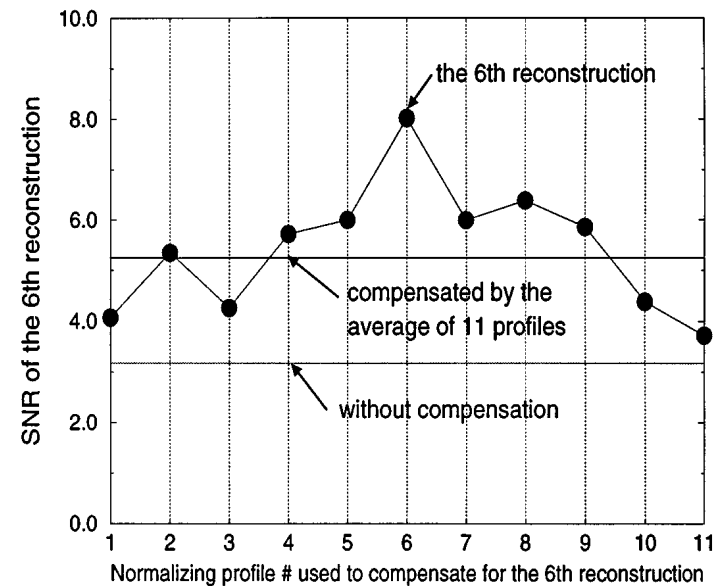

Fig. 6. SNR of the sixth reconstruction with different normalizing profiles.

recorded at the first null of the angular selectivity curve), development of interpixel gratings/fanning over the long exposure sequence, and nonuniform erasure of the recorded holograms owing to absorption in the crystal.

This normalization technique can be a useful method for improving performance in practice if a single normalizing profile can be used for all stored images. In Fig. 6 we display the SNR of the sixth reconstructed chessboard pattern with the normalization performed by each of the 11 normalizing profiles. The SNR drops off as the image is normalized with a normalizing profile collected at a reference beam angle that is increasingly different. This is due to the fact that the illuminated volume (by both the signal and the reference beams) changes, and crystal surface imperfections cause the variations in the normalization profile. A high-quality crystal and/or a recording geometry with fixed volume can overcome this problem, and a single normalizing profile stored electronically can be used to normalize the data. Alternatively, one can derive a single normalizing profile by averaging data collected over the entire angular bandwidth of the memory. The SNR obtained by normalizing the sixth chessboard pattern with the averaged normalizing profile is also shown in Fig. 6. A similar SNR was obtained with any of the 11 chessboard patterns.

The normalization technique compensates deterministic errors that can in principle be eliminated by care- ful design (e.g., antireflection coating the crystal and making the illumination more uniform). The residual errors are related to sources that are not easily eliminated (e.g., cross talk, interpixel gratings, fanning, detector noise, and media scatter noise). Therefore, if we use the normalized data to measure the probability of error, we obtain a rough estimate of the performance that we can expect in a carefully engineered holographic memory. Experimentally we detected an average of 19 errors in each of the 11 normalized images, corresponding to an estimate for the probability of error of $19 / 252,000=7.54 \times 10^{-5}$. The threshold level used to classify the pixels was optimized separately for each of the 11 cases. One of the reconstructions had no errors, whereas another had 68 errors. Two of the reconstructions had 104 of the 209 total errors. Many of the errors tend to cluster, appearing as small black spots at the same locations on every image, suggesting that imperfections such as dust particles on the SLM or the lenses are still a major source of errors in the normalized data. We can eliminate these errors by making the optical system cleaner than our laboratory environment. Therefore it is likely that, in a practical system, the probability of error can be somewhat less than $7.54 \times 10^{-5}$, the value obtained in our experiment.

We thank Geoffrey Burr and George Barbastathis for their helpful discussions and assistance with the data processing. This study was supported by the Rome Air Development Center and the U.S. Air Force Office of Scientific Research.

\section{References}

1. F. H. Mok, Opt. Lett. 18, 915 (1993).

2. G. W. Burr, F. H. Mok, and D. Psaltis, in Annual Meeting, Vol. 16 of OSA Technical Digest Series (Optical Society of America, Washington, D.C., 1993), paper TuHG.

3. L. D'Auria, J. P. Huignard, and E. Spitz, IEEE Trans. Magn. 9, 83 (1973).

4. I. McMichael, W. Christian, J. Hong, T. Y. Chang, R. Neurgaonkar, and M. Khoshnevisan, in Nonlinear Optics: Materials, Fundamentals, and Applications (Institute of Electrical and Electronics Engineers, New York, 1994).

5. S. Campbell, X. Yi, and P. Yeh, Opt. Lett. 19, 2161 (1994). 